\title{
Support for a white dwarf in the Be star binary $\gamma$ Cassiopeiae
}

\author{
K. M. V. Apparao* \\ 34, Vibha, opp. Cardinal School, R.P. Road, Bandra East, Bombay 400051, India \\ Received 12 October 2001 / Accepted 6 November 2001
}

\begin{abstract}
The recently discovered orbital period for $\gamma$ Cas and the modulation period in X-rays and optical are consistent with the orbital period-spin period relation for white dwarfs derived from a similar relation found for Be star - neutron star binaries. This lends support for the existence of a white dwarf in the $\gamma$ Cas binary.
\end{abstract}

Key words. stars: $\gamma$ Cas - white dwarf - stars: emission line, Be stars - stars: binaries - stars: neutron

\section{Introduction}

$\gamma$ Cas is a Be star of spectral type B0.5IV. X-ray emission was observed (see Apparao 1994a for references) with a luminosity of $\sim 10^{33} \mathrm{ergs} \mathrm{s}^{-1}$. Intensity variations and flaring in X-rays were observed (Parmar et al. 1993). The high temperature required for the $\mathrm{X}$-ray emission and $\mathrm{X}$-ray brightness (see Marlborough 1977) prompted a binary hypothesis for the system, with the X-ray emission occuring from accretion onto a compact object (white dwarf or neutron star).

Harmanec et al. (2000), using radial velocity variations of the $\mathrm{H} \alpha$ line have established the binary nature of $\gamma$ Cas. They find an orbital period $P_{\mathrm{d}}=203.59 \pm 0.29$ and an eccentricity $e=0.26$. Smith et al. (1998) found simultaneous but anticorrelated X-ray and UV modulations with a period 1.123. Harmanec (1999), using the Hipparcos satellite $H_{p}$ photometry in the optical, found a period 1. $15655 \pm 0.00012$. Robinson \& Smith (2000) using the RXTE satellite X-ray observation obtained a period 1.12277. Harmanec et al. (2000) reanalyzed the Hipparcos data and gave a best period of $1.48700 \pm 0.00013$. It seems that more work is needed in order to establish whether there are two distinct periods in the X-ray and optical as given above, possibly related, or if they are the same. Smith et al. (1998) and Robinson \& Smith (2000) suggest that the modulation period is the rotation period of the Be star while Harmanec et al. (2000) suggest that it could be either a corotation period or a low order mode of pulsation.

* e-mail: aprao@tifr.res.in

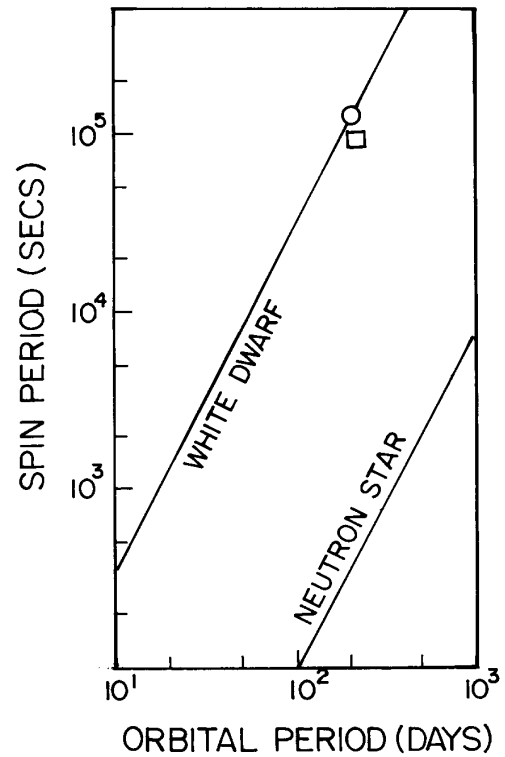

Fig. 1. The orbital period-spin period diagram for Be star binaries. The lines for neutron stars and white dwarfs are from Apparao (1994b). The square is for the modulation period derived by Robinson \& Smith (2000), while the circle is for the modulation period derived by Harmanec et al. (1999).

\section{The spin period-orbital period relation in Be star binaries}

Corbet $(1984,1986)$ found that a correlation exists between the spin period $P_{\mathrm{s}}$ and the orbital period $P_{\mathrm{o}}$ in $\mathrm{Be}$ star binaries. The basis of the relation is set out by Corbet (1984) and Waters \& van Kerkwijk (1989). The relation given by Corbet (1986) is for neutron stars and with a radius $R_{\mathrm{N}}=10^{6} \mathrm{~cm}$ and magnetic field $B_{\mathrm{N}}=10^{12} \mathrm{G}$, relation is shown in Fig. 1. Apparao (1994b), using the expressions for the relation given by Corbet (1986) and 
Waters \& van Kerkwijk (1989), obtained a similar relation for white dwarf stars in Be star binaries. The line shown for white dwarfs in Fig. 1 is for a radius $R_{\mathrm{WD}}=10^{9} \mathrm{~cm}$ and a surface magnetic field $B_{\mathrm{WD}}=10^{6} \mathrm{G}$.

As mentioned earlier, the nature of the periodicity of $\sim 1$ day is not established. However it is interesting if the period is attributed to the spin period of a white dwarf companion to the Be star and we can find its relationship with the orbital period. The points corresponding to the two periods $P_{\mathrm{s}}=1.12277$ (Robinson \& Smith 2000) and $P_{\mathrm{s}}=1.487$ (Harmanec et al. 2000) and the orbital period of 203.59 are plotted in Fig. 1.

\section{Discussion}

It is interesting to note that the points corresponding to the orbital period of $\gamma$ Cas and the $\sim 1$ day period fall on the line attributed to a white dwarf in the spin periodorbital period relation in Be stars. This lends support to the hypothesis that the compact object in the binary system is a white dwarf.

Kubo et al. (1998) argue that $\gamma$ Cas contains a white dwarf, while Robinson \& Smith (2000) suggest that the observed phenomena in X-rays and UV can be explained as being due to processes near the primary Be star. In the following, we will discuss their arguments and give our comments: i) The X-ray luminosity of $\gamma$ Cas is $\sim 10^{33} \mathrm{ergs} \mathrm{s}^{-1}$. This is higher than what is expected of an early type B-star. Kubo et al. (1998) suggest that the $\mathrm{X}$-ray emission is from a white dwarf companion to the Be star. They assume Bondi-Hoyle type of accretion from a "wind" emanating from the Be star. Using a wind velocity of $200 \mathrm{~km} \mathrm{~s}^{-1}$, they obtain the requisite X-ray luminosity. Robinson \& Smith (2000) argue that Kubo et al. have not taken into account the efficiency of conversion of the accretion energy into X-ray, which is less than one and therefore cannot acheive the requisite luminosity. However, as Waters et al. (2000) have suggested, Be star disks are Keplerian with very small radial velocities (see also Apparao 1985). The orbital motion of the compact object, while it is immersed in the gas disk, is also Keplerian. Consequently, the relative velocity between the white dwarf and the gas in the disk is very small, resulting in large accretion rates. This is shown by the large accretion rate needed to explain the super-Eddington X-ray luminosity of the system A0538-66 and also the high luminosity of many Be star-neutron star systems (see Apparao 1994a). Thus the accretion rates needed to explain the X-ray luminosity of $\gamma$ Cas can be acheived. ii) Smith et al. (1994) and Smith \& Robinson (2000) observed flares and rapid flickering in the X-ray emission of $\gamma$ Cas. They suggested that the observed phenomena can be explained by processes near the Be star. Kubo et al. (1998) have argued that similar flaring and flickering occurs in the X-ray emission of SS Cygni, which contains an accreting white dwarf. Smith \& Robinson (2000) point out that SS Cygni is a close binary and the accretion process is different from that in $\gamma$ Cas. However flares can occur if the white dwarf passes through clumps of gas that may be present in the gas disk.

Smith \& Robinson (2000) have also observed an anticorrelation in the intensities of X-ray and UV emissions. Such anti-correlation might result if an increased X-ray emission is due to increased density of gas in the disk, which can cause a decrease in the UV luminosity.

The detection of the white dwarf in the $\gamma$ Cas system directly is difficult. Cyclotron lines have been observed in magnetic white dwarfs (Wickramasinghe 1982) in the optical and UV regions. However, these lines will not be visible due to the higher luminosity of the Be star in this region. Accreting magnetic white dwarfs give a luminosity in the far UV and soft X-ray region a factor 10-100 larger than the hard X-ray luminosity (Kylafis \& Lamb 1982; Mason et al. 1978). The effects due to this higher luminosity may be detectable. This aspect will be discussed in a future publication.

It is clear that further work is needed to understand the phenomena observed in $\gamma$ Cas. However, our observation that the orbital period and the $\sim 1$ day period in $\gamma$ Cas are consistent with the orbital period-spin period relation expected from Be star-white dwarf binaries, lends support for a white dwarf companion to the Be star in $\gamma$ Cas.

Acknowledgements. I thank the Director of Tata Institute of Fundamental Research for allowing me the use of their Library and other facilities for this research. I thank Prof. P. C. Agrawal for discussions.

\section{References}

Apparao, K. M. V. 1985, ApJ, 292, 257

Apparao, K. M. V. 1994a, SpSc Rev., 69, 255

Apparao, K. M. V. 1994b, A\&A, 291, 775

Corbet, R. H. D. 1984, A\&A, 141, 91

Corbet, R. H. D. 1986, MNRAS, 220, 1047

Harmanec, P. 1999, A\&A, 341, 867

Harmanec, P., Habuda, P., Stefl, S., et al. 2000, A\&A, 364, L85

Kubo, S., Murakami, T., Ishida, M., \& Corbet, R. H. D. 1998, PASJ, 50, 417

Kylafis, N. D., \& Lamb, D. Q. 1982, ApJ, 48, 239

Marlborough, J. M. 1977, PASP, 89, 122

Mason, K. O., Lampton, M., Charles, P., \& Bowyer, P. 1978, ApJ, 226, L129

Parmar, A.,Israel, G., Stell, L., \& White, N. 1993, A\&A, 275, 227

Robinson, R. D., \& Smith, M. A. 2000, ApJ, 540, 474

Smith, M. A., Robinson, R. D., \& Corbet, R. H. D. 1998, ApJ, 503,877

Waters, L. B. F. M., \& van Kerkwijk, M. H. 1989, A\&A, 223, 196

Waters, L. B. F. M., et al. 2000, IAU Colloq. 175, The Be Phenomenon and Early Type Stars, ed. M. A. Smith, H. Heinrichs, \& J. Fabregat, in press

Wickramasinghe, D. T. 1982, Proc. ASA, 4(4) 